\title{
Non-Destructive Identification of Fibre Orientation in Multi-Ply Biaxial Laminates Using Contact Temperature Sensors
}

\author{
David I. Gillespie ${ }^{1,2, * \mathbb{C}}$, Andrew W. Hamilton ${ }^{1}\left(\mathbb{D}\right.$, Ewan J. McKay ${ }^{2}$, Brian Neilson ${ }^{2}$, \\ Robert C. Atkinson ${ }^{1}$ (D), Ivan Andonovic ${ }^{1}$ (D) and Christos Tachtatzis ${ }^{1}$ (D) \\ 1 Department of Electronic and Electrical Engineering, University of Strathclyde, Royal College Building, \\ 204 George Street, Glasgow G1 1XW, UK; andrew.w.hamilton@strath.ac.uk (A.W.H.); \\ robert.atkinson@strath.ac.uk (R.C.A.); i.andonovic@strath.ac.uk (I.A.); christos.tachtatzis@strath.ac.uk (C.T.) \\ 2 Collins Aerospace, Prestwick, 1 Dow Avenue, Prestwick International Aerospace Park, \\ Ayrshire KA9 2SA, UK; ewan.mckay@collins.com (E.J.M.); Brian.neilson@collins.com (B.N.) \\ * Correspondence: david.gillespie@strath.ac.uk
}

Received: 5 June 2020; Accepted: 8 July 2020; Published: 10 July 2020

check for updates

\begin{abstract}
Fibre orientation within composite structures dictates the material properties of the laminate once cured. The ability to accurately and automatically assess fibre orientation of composite parts is a significant enabler in the goal to optimise the established processes within aftermarket aerospace industries. Incorrect ply lay-up results in a structure with undesirable material properties and as such, has the potential to fail under safe working loads. Since it is necessary to assure structural integrity during re-manufacture and repair assessment, the paper demonstrates a novel method of readily and non-destructively determining fibre orientation throughout multi-ply Biaxial woven composite laminates using point temperature contact sensors and data analysis techniques. Once cured, only the outermost laminates are visible to assess orientation. The inspection method is conducted visually, with reference guides to allow for rapid adoption with minimum training, as well as harnessing established temperature sensors within the Maintenance Repair and Overhaul (MRO) environment. The system is amenable to integration within existing repair/re-manufacture processes without significant impact to process flow. The method is able to identify noisy samples with an accuracy, precision and recall of 0.9 , and for synthetically created samples of double the cure ply thickness, a precision of 0.75 , a recall of 0.7 and an accuracy of 0.87 .
\end{abstract}

Keywords: aerospace; composite; inspection; maintenance repair overhaul; non destructive inspection; quality assurance; re-manufacture; thermography

\section{Introduction}

Low cost temperature sensors and low temperature heat sources form the basis for the implementation of a method to identify differences in thermal profiles of composites from low resolution data capture [1]. Within the Maintenance Repair and Overhaul (MRO) aerospace operational environment, the demand is not only to provide quality assurance of executed repairs, but to identify and characterise repairs executed by third parties for which there are often partial or no supporting records. The repair of composite structures requires removal of the damage and surrounding areas via cutting and drilling. The optimal performance of machine tooling used in processing Carbon Fibre Reinforced Plastic (CFRP) is application specific governed by the fibre characteristics within the structure [2].

The fibre orientation within a composite part has an impact upon the failure rate of Fibre Reinforced Plastic (FRP) laminate structures undergoing cyclic loading [3]. Non-symmetrical ply 
lay-ups create cured laminates with deformations that vary with fibre orientation symmetry [4-6]. In thin laminates, deformations are visible due to the magnitude of strain within the structure. Stiffness of the laminate- the resistance to bending - is increased by thickening the structure [7] or through the creation of a sandwich of two laminates bonded to either side of a stiffener, such as a honeycomb core [8]. Consequently visual inspection for bending due to the symmetry of laminate plies on thick and sandwich composite structures are unlikely to indicate non-symmetrical lay-ups.

X-ray computed tomography is an industry standard technique-amenable to enhancements in terms of information content through machine learning algorithms-that identifies individual fibre orientations within laminate structures and provides an indication as to the mean orientation of the laminate [9-11]. Results can be obtained quickly with a high degree of accuracy and level of detail for samples within the scan area. However for larger structures, several images are required to be stitched together to produce a full scan. Owing to the safety consideration associated with the use of $\mathrm{X}$-rays in respect to human operators, the inspection equipment is ideally automated with either (i) static samples and moving scanners, (ii) static scanners and moving samples or (iii) a hybrid, the use of X-ray computational tomography becomes a time consuming and expensive process. The MRO environment differs to that of an Original Equipment Manufacturer (OEM), especially in respect to the nature of the structure condition. An alternative inspection method is Infra Red (IR) Thermography, it is well established that IR Thermography is able to provide accurate and reliable indications of fibre orientation [12-18]; however, Infrared Thermogrphic techniques rely on a consistent surface emissivity [19]. OEM parts are generally constructed from new materials and as such are clean and free from contaminants. MRO structures originate from in-service aircraft and are often subject to differing degrees of repair, including modifications to the surface e.g., paint removal and surface treatments. As such this technique produces variable results under these conditions.

A modelling approach to characterising the thermal profile of Biaxial laminates and identify ply orientation, is to rely entirely on finite element analysis. However, reported research has demonstrated that the transient thermal interactions are more complex at the ply boundaries and when simplified the resultant models are limited [1].In particular, transient thermal simulations through finite element analysis concluded that two ply Biaxial laminate samples produce a balanced thermal spread in the $x$ - and $y$-axis along the length of the fibres for $0 / 90 / 90 / 0$ and $0 / 90 / 0 / 90$ samples [1]. Changes of 90 degrees to the fibre orientation at this boundary result in a significant change to the thermal profile on the far side of a sample in terms of thermal transmission. Contact temperature sensors for cure monitoring such as thermocouples and Resistance Temperature Detectors (RTDs) are in routine use in composite repair processes and as such the devices, configuration and measurements are well understood and accepted by operators. The proposed approach will utilise existing cure monitoring sensors and heat sources in order to generate thermal profile data, adopting the principles of traditional IR thermography but implemented through contact temperature sensors.

As the existing range of techniques, which can accurately and reliably produce indications of fibre orientation, currently require equipment and training that is not immediately available within most MRO facilities, there is a financial motivation for the need of a new method to inspect composite structures utilising existing equipment with minimal training. The method makes use of low cost contact temperature sensors and low temperature heater mats, currently used in the composite repair process within MROs, to identify fibre orientation within the repair regions of composite parts. This enables aerospace MRO providers to deliver services in a safer, faster and scalable manner.

\section{Materials and Methods}

In total, 3 Biaxial woven aerospace-grade five harness CFRP pre-impregnated laminates were produced in $500 \times 500 \mathrm{~mm}$ samples to prove the feasibility of the method. Each laminate comprised two ply samples of $1589 \mathrm{~kg} \mathrm{~m}^{-1}$ density and the thermal properties described in Table 1 . These were laid up with three differing fibre orientations; 0/90/90/0 (symmetrical and balanced); 0/90/0/90 (non-symmetrical and balanced); and $0 / 90 /+45 /-45$ (non-symmetrical and unbalanced). The tool 
to obtain thermal conduction profiles across the samples comprised two elements; 64 Resistance Temperature Detector (RTD) array with sensors spaced at $37.5 \mathrm{~mm}$ intervals within a $300 \times 300 \mathrm{~mm}$ grid; and a $150 \times 150 \mathrm{~mm}$ Proportional Integral Derivative (PID) controlled heat mat (Figure 1). The heat mat was applied to the bottom surface of the sample under inspection and the RTD probe array applied to the top surface. The samples were orientated such that the top surface in contact with the RTD array displayed the $0 / 90$ fibre orientation, with the rear surface of the sample in contact with the heat mat varying between $0 / 90,90 / 0$ and $+45 /-45$ depending on the sample.

Table 1. Material thermal properties for biaxial laminate composite plies.

\begin{tabular}{|c|c|c|c|c|}
\hline $\begin{array}{c}\text { Temperature } \\
\text { (K) }\end{array}$ & $\begin{array}{c}\text { Thermal } \\
\text { Conductance } \\
\text { X Direction } \\
\left(\mathbf{W} \mathbf{m}^{-1} \mathbf{K}^{-1}\right)\end{array}$ & 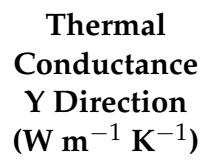 & 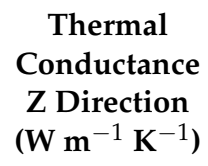 & $\begin{array}{c}\text { Specific } \\
\text { Heat } \\
\left(\mathrm{J} \mathrm{kg}^{-1} \mathrm{~K}\right)\end{array}$ \\
\hline 218.15 & 9.3287 & 9.3287 & 0.6923 & 678.26 \\
\hline 295.93 & 11.198 & 11.198 & 0.9346 & 904.35 \\
\hline 394.26 & 12.981 & 12.981 & 1.0384 & 1184.9 \\
\hline
\end{tabular}

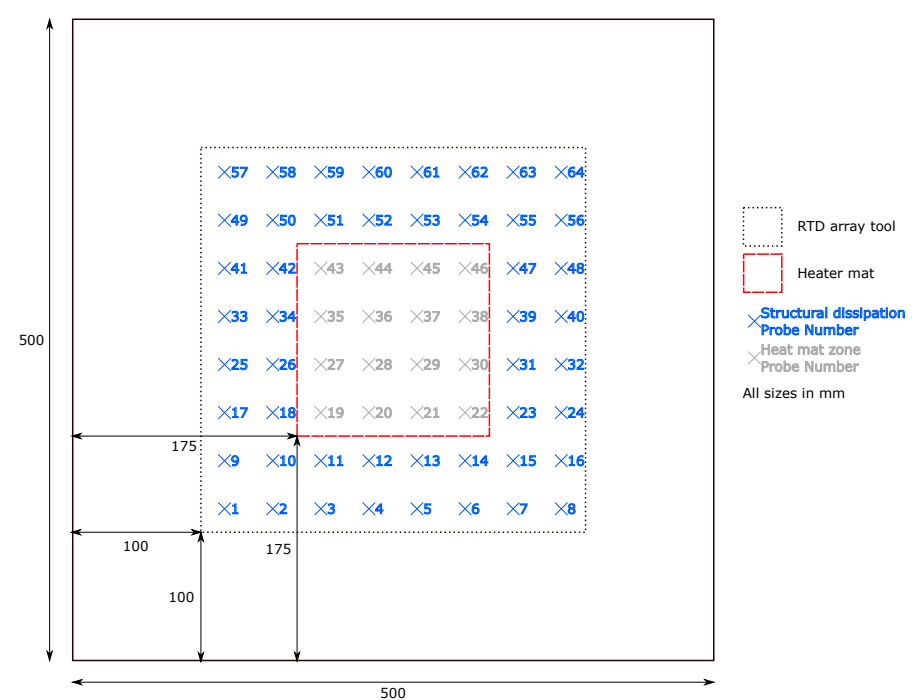

Figure 1. The 500 by $500 \mathrm{~mm}$ sample panel data capture set up with $150 \mathrm{~mm} \times 150 \mathrm{~mm}$ heat zone (dashed red) and 300 by $300 \mathrm{~mm}$ RTD probe array (dashed black).

The thermal stimulus following a step profile within the heat zone (Figure 1) is outlined in Table 2. The stimulus period was 5 minutes to allow sufficient time for the transient thermal profile to develop and the thermal energy to conduct and dissipate.

Table 2. Step heating profile for heat zone set up.

\begin{tabular}{ccccc}
\hline Stage & $\begin{array}{c}\text { Time Step } \\
\text { (s) }\end{array}$ & $\begin{array}{c}\text { Start } \\
\text { Temperature (K) }\end{array}$ & $\begin{array}{c}\text { End } \\
\text { Temperature (K) }\end{array}$ & $\begin{array}{c}\text { Ramp } \\
\text { Rate (K/s) }\end{array}$ \\
\hline Ramp & $0-100$ & 298.45 & 353.15 & 0.55 \\
Dwell & $100-300$ & 353.15 & 353.15 & 0.00 \\
\hline
\end{tabular}

The temperature recorded on the top surface of the laminate can be attributed to two key thermal transfer processes. The area directly above the heat zone is the result of thermal transmission though the laminate, where as areas outside the heated zone experience temperature changes due to the compounding effect of transmission and thermal conduction in the direction of the fibres. The temperature change $(\Delta T)$ was recorded over time $(t)$ for all temperature probes. The probes positioned directly above the heat zone do not show significant variations between samples that 
permit the discrimination of ply lay-ups. However, probes positioned in the the areas outwith the heat zone are primarily impacted by thermal conduction through the fibres and consequently permit the differentiation of fibre orientation and ply lay-up.

\section{Analysis of Results}

Initial analysis of the samples was conducted through heat maps to identify significant visual differences in the thermal profile. Variation over the heated area is evident on comparison of all three samples at corresponding time intervals (see Figure 2). However such variations that may be observed produce patterns which are subjective and as such, are not sufficiently robust as an inspection method alone. Furthermore, no readily apparent differences are visible between maps outwith the heat zone and temperature changes within the dissipation area are attributed to the thermal conduction along the fibres. However, from observation, the thermal spread is governed by the fibre directions, manifest by the temperature changes $(\Delta T)$ outwith the heated area varying between $0{ }^{\circ} \mathrm{C}$ and approximately $12{ }^{\circ} \mathrm{C}$. Consequently further analysis will concentrate on the regions where the temperature differences fall within that range highlighted in blue on Figure 1.
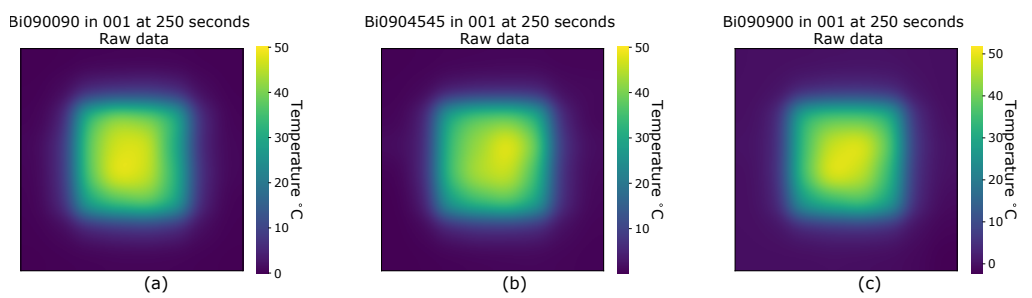

Figure 2. Heat maps of samples at $80^{\circ} \mathrm{C}$ and 250 seconds, (a) Biaxial 0/90/0/90, non symmetrical and balanced, (b) Biaxial 0/90/+45/-45, non symmetrical and unbalanced, (c) Biaxial 0/90/90/0, symmetrical and balanced.

A Kernel Density Estimate (KDE) was used to generate the probability density function as a function of temperature change in ${ }^{\circ} \mathrm{C}$ and to present the data in a more visually descriptive manner, easing the initial inspection. KDE was selected due to the ability to provide readily interpreted visualisation of quantitative data [20]. A bandwidth of 1 , selected through trial and error, was used to achieve a constant bandwidth between samples that prevented over-smoothing of the data and allowed the accentuation of features.

Figure 3a-c shows the $\Delta T$ distribution Biaxial samples with lay-ups of 0/90/0/90, 0/90/ $+45 /-45$ and 0/90/90/0 respectively. An overall KDE and its median KDE is computed for every second in the 150-300 time window for every sample (the solid line). The multi coloured region is a side view of the tri-surface plot of the density spread of individual samples as shown in Figure 4.

From Figure $3 a$ the balanced non-symmetrical samples of 0/90/0/90 show a bi-modal distribution of temperature change which can be attributed to the fibre orientation interfaces between plies. For the second group of samples, unbalanced non-symmetrical $0 / 90 /+45 /-45$, the transitions in fibre orientations are not as steep and consequently the second mode is attenuated. Finally, for the last group of samples, balanced symmetrical 0/90/90/0, the transition between the two slopes is even less steep than that of the unbalanced non-symmetrical $0 / 90 /+45 /-45$. The significant feature of the KDE characteristic is the presence of two clear peaks evolving by the 300 second timestamp for all of the sample runs. The visualisation of the incremental KDE for each fibre orientation of one sample, accentuates the transitions over time. Figure $4 \mathrm{a}-\mathrm{c}$ displays KDE plots at between 150 and $300 \mathrm{~s}$ for one sample of Biaxial 0/90/0/90, Biaxial 0/90/ $+45 /-45$ and 0/90/90/0 respectively. The transition into two distinct peaks for Biaxial 0/90/ $+45 /-45$ and Biaxial 0/90/90/0 (Figure $4 \mathrm{~b}, \mathrm{c}$ ) becomes more evident. The KDE for Biaxial 0/90/0/90 balanced and non-symmetrical in Figure 4a displays two distinct peaks from $150 \mathrm{~s}$ to $300 \mathrm{~s}$, with the transition of the secondary peak advancing along the Temperature Range $\mathrm{C}$ axis ( $x$-axis). 

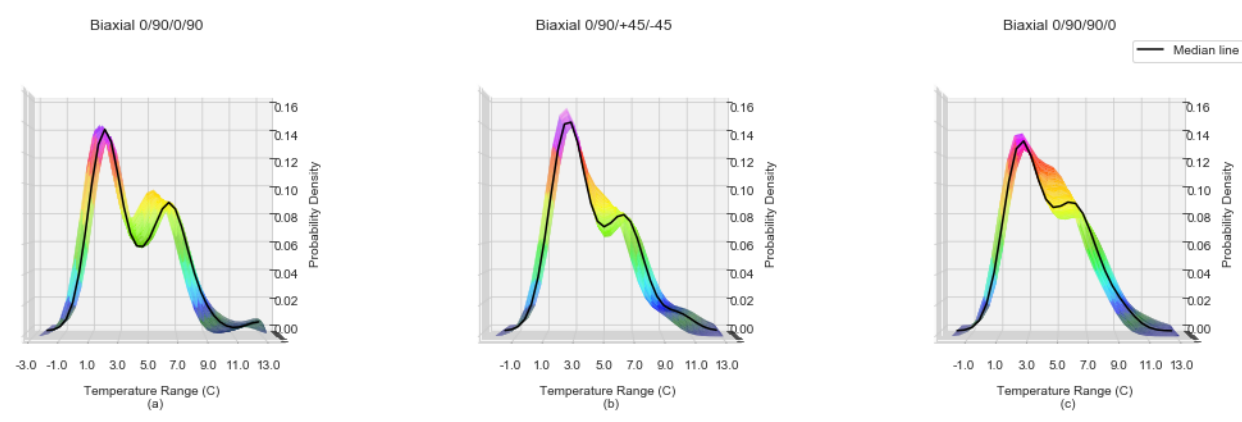

Figure 3. Kernel density estimates with median line for Biaxial laminate samples for temperature changes ranges $-3^{\circ}$ to $12^{\circ}$ for the time period 150 to $300 \mathrm{~s}$ (a) Biaxial 0/90/0/90, non symmetrical and balanced, (b) Biaxial 0/90/+45/-45, non symmetrical and unbalanced, (c) Biaxial 0/90/90/0, symmetrical and balanced.

Biaxial 0/90//90

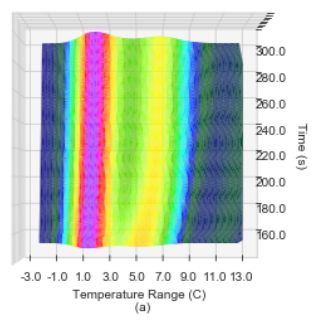

Biaxial 0/90/+45/-45

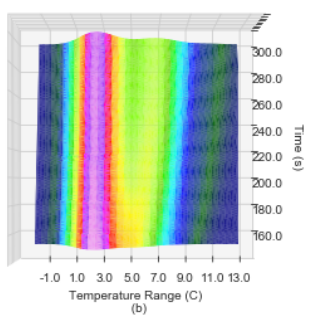

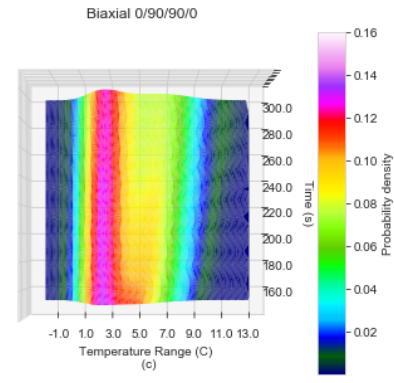

Figure 4. Kernel density estimates top down view of laminate samples for temperature changes ranges $-3^{\circ}$ to $12^{\circ}$ for the time period 150 to $300 \mathrm{~s}$ (a) Biaxial 0/90/0/90, non symmetrical and balanced, (b) Biaxial 0/90/+45/-45, non symmetrical and unbalanced, (c) Biaxial 0/90/90/0, symmetrical and balanced.

The transition of the bi-modal peaks forming within the differing fibre orientation sample types becomes clear on inspection of the tri-surface plot of all KDE points as a function of time within a 3D plot viewed from above as shown in Figure 4. Figure 4a displays the Biaxial 0/90/0/90 balanced and non-symmetrical sample with two distinct peaks from the start of 150 second time frame. The peaks settle into perpendicular alignment by approximately the $220 \mathrm{~s}$. Within the Biaxial 0/90/+45/ - 45 unbalanced sample shown in Figure $4 \mathrm{~b}$ the secondary peak appears to form the $200 \mathrm{~s}$ and a perpendicular alignment of the bi-modal peaks does not become apparent until the $240 \mathrm{~s}$. The fibre orientation sample type of Biaxial 0/90/90/0 displays two peaks which remain within close proximity of each other in regards to the $z$-axis (probability density) which do not appear to form a perpendicular alignment within the 150-300 second time period.

\section{Data Preparation and Comparative Analysis}

In order to provide direct comparative analysis, plots were generated between $-2{ }^{\circ} \mathrm{C}$ and $13{ }^{\circ} \mathrm{C}$ at intervals of $0.25{ }^{\circ} \mathrm{C}$, interpolated from the generated $\mathrm{KDE}$ plot points via the numpy function (numpy.interp) for all samples. The interpolated points were then used to create KDE as a function of time plots in the same style as in Figure 4. The variation between the $y$ axis (Probability Density) can be compared since these plots are created at a fixed $x$-axis (Temperature Range) and $z$-axis (Time (s)) point. The Mean Square Error (MSE) between two samples can also be evaluated as the interpolation creates a 3 dimensional array. The expectation is that when comparing a randomly selected sample to three samples of differing fibre orientation, the lowest MSE would most likely indicate the samples that share the same fibre orientation. To test the hypothesis, the 30 data sets created from the 10 runs of 
each of the three fibre orientation samples were split randomly into two groups; the first contained $40 \%$ of the total sample data sets used as the testing group; the remaining $60 \%$ split further into three groups by fibre orientations. The fibre orientation sets were used to create a mean baseline data set for each fibre orientation by generating a mean $\mathrm{KDE}$ as a function of time plot. $(\overline{K D E} / t)$. The test group data sets were then compared individually against the $\overline{K D E} / t$ of each fibre orientation and ranked green, amber and red, with green designating the lowest and red the highest MSE; the classification approach utilised in the visual representation of each $\overline{K D E} / t$.

An example of the representation is shown in Figure $5 b$ where the MSE is the lowest of the three and is thus coloured green, a true positive classification as this is the $\overline{K D E} / t$ generated for Biaxial $0 / 90 /+45 /-45$. It is also clear on visual inspection that the median of the test sample of unknown fibre orientation (shown in black) sits completely in the $\overline{K D E} / t$ of Figure $5 \mathrm{~b}$. In order to quantify the effectiveness of the method the predicted results that the method generated were plotted against the actual values in a confusion matrix (Figure 6). The confusion matrix is traditionally used within machine learning to assess machine learning algorithms ability to correctly predict known test data against training data [21]. As the confusion matrix is designed to present how a Machine Learning algorithm is performing by plotting the predicted values in a grid format against the actual values this method allows for quick visual confirmation as to the performance of the method as well as any increase or decrease between modifications to the method. Within the confusion matrix four classification types can be produced;

- True Positive;

- True Negative;

- False Positive;

- $\quad$ False Negative.
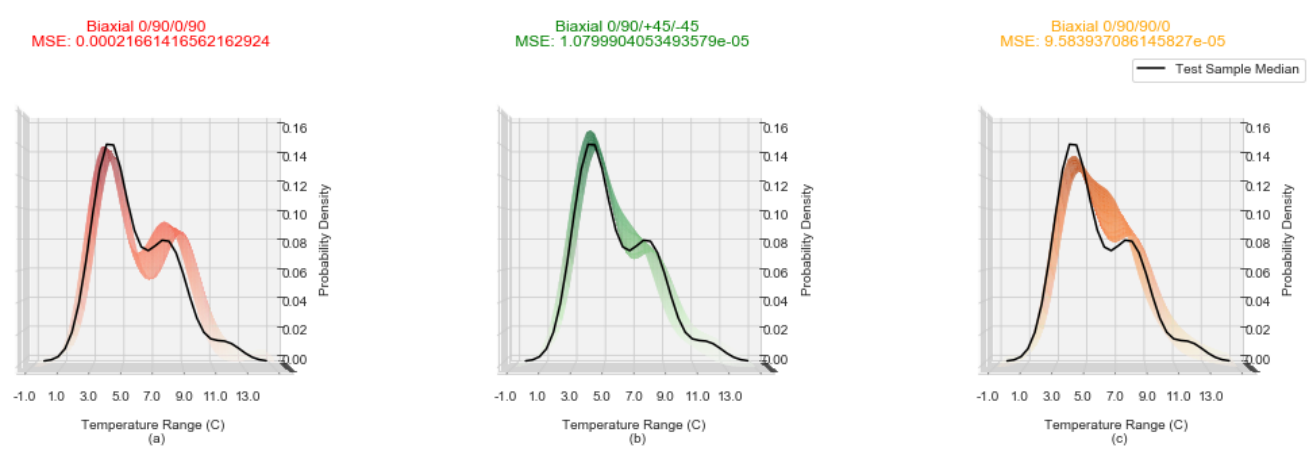

Figure 5. A Biaxial 0/90/ $+45 /-45$ sample compared against the $\overline{K D E} / t$ for (a) Biaxial 0/90/0/90, (b) Biaxial 0/90/+45/-45, (c) Biaxial 0/90/90/0.

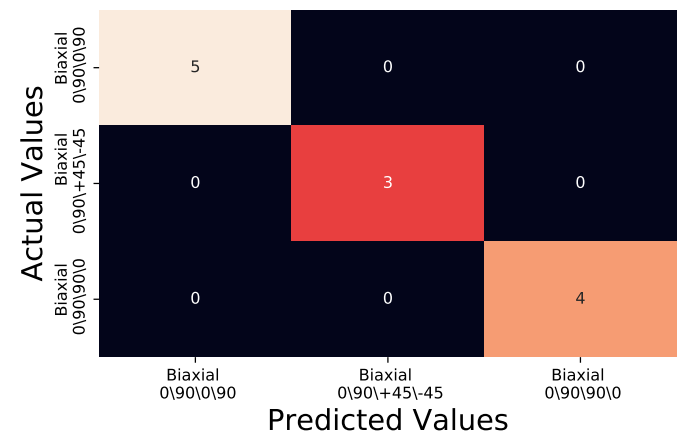

Figure 6. Confusion matrix for testing group samples MSE when compared to $\overline{K D E} / t$. 
For the Biaxial 0/90/0/90 samples a true positive would be a correct predicted value of Biaxial 0/90/0/90 against the actual value of Biaxial 0/90/0/90. A true negative would be either Biaxial $0 / 90 /+45 /-45$ predicted value against actual value Biaxial 0/90/ $+45 /-45$ or Biaxial 0/90/90/0 predicted value against actual value Biaxial 0/90/90/0. False positives in the case of Biaxial 0/90/0/90 come in the form of predicted values of Biaxial 0/90/ $+45 /-45$ or Biaxial 0/90/90/0 against actual values for Biaxial 0/90/0/90. Finally a false negative value for Biaxial 0/90/90/0 is the instance that predicted values for Biaxial 0/90/0/90 are against either Biaxial 0/90/ $+45 /-45$ or Biaxial 0/90/90/0 actual values. These classification types are transferable to the other two sample fibre orientation types where Biaxial 0/90/0/90 is swapped with the fibre orientation of which the classification type is being identified.

Figure 6 shows that the small selection of test samples are all correctly classified with their correct fibre orientation, this is seen by the overlap of predicted values and actual values being $100 \%$. Further to the visual display of the confusion matrix, the results can be further analysed to produce precision, recall, F1 scores and overall accuracy of the results. By creating a ratio of predicted values which were correctly identified as the actual values the precision of the method can be quantified (1).

$$
\text { Precision }=\frac{\text { TruePositive }}{\text { TruePositive }+ \text { FalsePositive }}
$$

The recall of the results is a quantification of how many of the actual values did the predicted values label as such (2).

$$
\text { Recall }=\frac{\text { TruePositive }}{\text { TruePositive }+ \text { FalseNegative }}
$$

An F1 score can also be produced, which is a weighted average of the already calculated Precision and Recall (3). Should the method be tested with an uneven distribution of fibre orientations between test samples the F1 score provides a good indication of comparative performance between test samples.

$$
F 1=2 * \frac{\text { Recall } * \text { Precision }}{\text { Recall }+ \text { Precision }}
$$

Finally the overall accuracy of the method based on the confusion matrix results, which is the ratio of all correct identifications against all values (4) can also be calculated which produces a quantitative value to compare the methods when changes are introduced.

$$
\text { Accuracy }=\frac{\text { TrueNegative }+ \text { TruePositive }}{\text { TrueNegative }+ \text { FalsePositive }+ \text { TruePositive }+ \text { FalseNegative }}
$$

As seen in Figure 6 only 12 samples MSE were created against the generated $\overline{K D E} / t$.

\section{Synthetic Data Inspection}

Due to the COVID19 pandemic of 2020, the authors were unable to obtain further experimentally captured sample readings than the 30 captured prior to restricted access to facilities. In order to produce a larger sample data set an additional six groups of synthetic data sets were generated from the original 30 to further test the accuracy and precision of the method. The data sets were created through the addition of random noise to the experimentally captured thermal profiles. Random Gaussian noise for bandwidths ranging from 0.1 to 0.6 in 0.1 increments were added to each probe reading per second to generate 1200 synthetic samples per bandwidth. To simulate the minor deviation observed in temperature readings between the experimentally captured data sets the random Gaussian noise bandwidth of 0.3 were seen to produce temperatures that fell within the temperature against time profiles of the experimentally captured data sets whilst still providing temperature against time profiles that differed from those experimentally captured. The synthetic data sets were subject to the same MSE comparison as the original experimentally captured test group, against the same $\overline{K D E} / t$ for each 
fibre orientation. Figure 7a-f displays the confusion matrices for these synthetic data sets; evident is the strong true positive identification of Biaxial 0/90/0/90 at all bandwidths, reaching 100\% precision and recall at bandwidth 0.3 continuing through to bandwidth 0.1 (Table 3). The lack of variation in the confusion matrix results between bandwidths $0.1,0.2$ and 0.3 (Figure $7 \mathrm{~d}-\mathrm{f}$ ) provide reassurance that the use of a bandwidth of 0.3 does provide a larger simulated data set compared to the experimentally captured data set without producing results that would differ excessively from those expected from experimentally captured data sets. The Biaxial 0/90/ $+45 /-45$ has the highest rate of false positives of the three fibre orientations, identifying 80, 74 and 42 samples respectively as Biaxial 0/90/90/0 for bandwidths 0.6, 0.5 and 0.4 respectively. Both Biaxial 0/90/+45/-45 and Biaxial 0/90/90/0 mirror false positives as each other for 40 samples each for bandwidths 0.3 to 0.1 , highlighted by identical recall and precision in Table 3 at bandwidths $0.3,0.2$ and 0.1 .

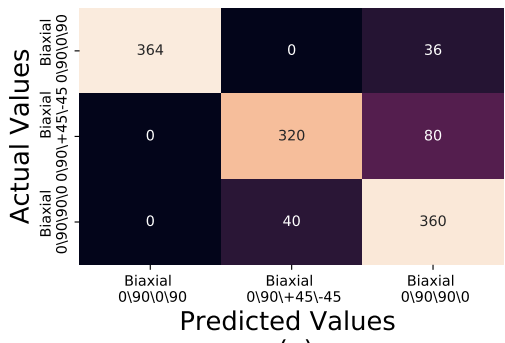

(a)

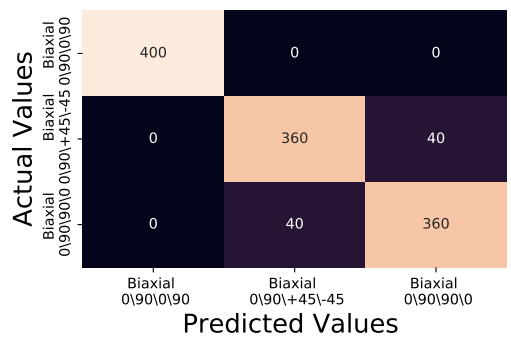

(d)

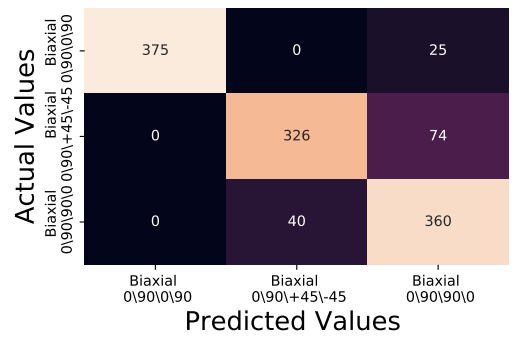

(b)

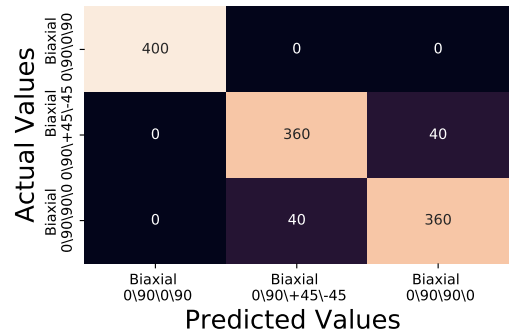

(e)

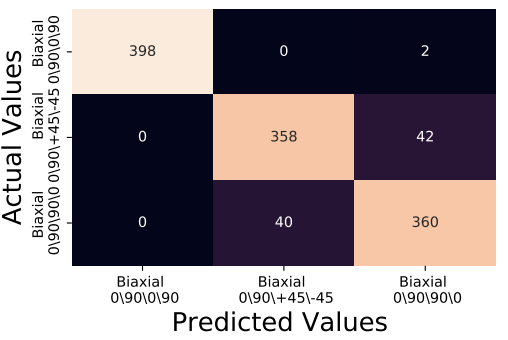

(c)

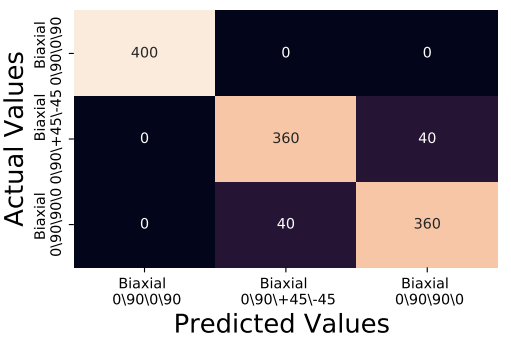

(f)

Figure 7. Confusion matrices of Biaxial 0/90/0/90, Biaxial 0/90/ $+45 /-45$ and Biaxial 0/90/90/0, for bandwidths: (a) 0.6 (b) 0.5 (c) 0.4 (d) 0.3 (e) 0.2 (f) 0.1 .

Table 3. Classification report for synthetic samples, bandwidths 0.6 through to 0.1 .

\begin{tabular}{|c|c|c|c|c|c|}
\hline Bandwidth & Sample & Precision & Recall & F1-Score & Accuracy \\
\hline \multirow{3}{*}{0.6} & Biaxial 0/90/0/90 & 1.00 & 0.91 & 0.95 & \multirow{3}{*}{0.87} \\
\hline & Biaxial 0/90/+45/-45 & 0.89 & 0.80 & 0.84 & \\
\hline & Biaxial 0/90/90/0 & 0.76 & 0.90 & 0.82 & \\
\hline \multirow{3}{*}{0.5} & Biaxial 0/90/0/90 & 1.00 & 0.94 & 0.97 & \multirow{3}{*}{0.88} \\
\hline & Biaxial 0/90/+45/-45 & 0.89 & 0.81 & 0.85 & \\
\hline & Biaxial 0/90/90/0 & 0.78 & 0.90 & 0.84 & \\
\hline \multirow{3}{*}{0.4} & Biaxial 0/90/0/90 & 1.00 & 0.99 & 1.00 & \multirow{3}{*}{0.93} \\
\hline & Biaxial 0/90/+45/-45 & 0.90 & 0.90 & 0.90 & \\
\hline & Biaxial 0/90/90/0 & 0.89 & 0.90 & 0.90 & \\
\hline \multirow{3}{*}{0.3} & Biaxial 0/90/0/90 & 1.00 & 1.00 & 1.00 & \multirow{3}{*}{0.93} \\
\hline & Biaxial 0/90/+45/-45 & 0.90 & 0.90 & 0.90 & \\
\hline & Biaxial 0/90/90/0 & 0.90 & 0.90 & 0.90 & \\
\hline \multirow{3}{*}{0.2} & Biaxial 0/90/0/90 & 1.00 & 1.00 & 1.00 & \multirow{3}{*}{0.93} \\
\hline & Biaxial 0/90/+45/-45 & 0.90 & 0.90 & 0.90 & \\
\hline & Biaxial 0/90/90/0 & 0.90 & 0.90 & 0.90 & \\
\hline \multirow{3}{*}{0.1} & Biaxial 0/90/0/90 & 1.00 & 1.00 & 1.00 & \multirow{3}{*}{0.93} \\
\hline & Biaxial 0/90/+45/-45 & 0.90 & 0.90 & 0.90 & \\
\hline & Biaxial 0/90/90/0 & 0.90 & 0.90 & 0.90 & \\
\hline
\end{tabular}


A further synthetic data set was created to simulate Biaxial laminates of double the thickness by extending the temperature against time response. The data were generated by inserting a blank time period between each second captured and interpolating the temperature at this time period from the previous and next temperature, in so doing artificially doubling the thermal response time and generating a thermal map representative of laminate plies double the thickness of the experimental samples. Noise from random points of a Gaussian normal process with bandwidth 0.1 was applied to generate 1200 double thickness data points. As before, the synthetic data set was subject to the same MSE comparison as the original experimentally captured test group, against the same $\overline{K D E} / t$ for each fibre orientation.

The results for Biaxial 0/90/0/90 and Biaxial 0/90/+45/-45 are comparable to those of the regular thickness samples at bandwidth 0.3 as shown in Figure 8 and Table 4 . However Biaxial $0 / 90 /+45 /-45$ has a reduction in precision from 0.9 to 0.5 , due to False Positive identification of the Biaxial 0/90/90/0 samples as Biaxial 0/90/+45/ -45 , also reflected within the precision and recall scores of Biaxial $0 / 90 / 90 / 0$ as 0.88 and 0.7 respectively. Overall, the accuracy between the normal thickness and the double thickness samples with random Gaussian normal noise applied at 0.1 bandwidth reduced from 0.93 in the regular to 0.87 in the double thickness samples.

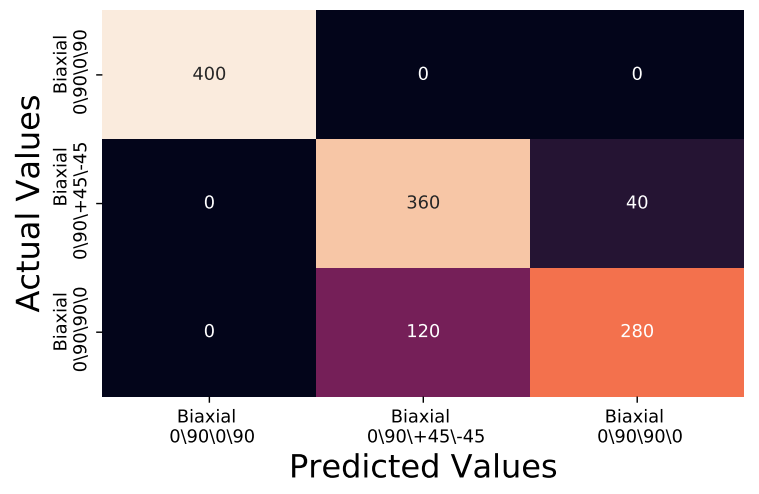

Figure 8. Confusion matrix for synthetically created double thickness samples with 0.1 bandwidth random point of Gaussian normal added for noise MSE when compared to $\overline{K D E} / t$.

Table 4. Classification report for double thickness synthetic sample, with addition of random Gaussian normal noise bandwidth 0.1 .

\begin{tabular}{cccccc}
\hline Bandwidth & Sample & Precision & Recall & F1-Score & Accuracy \\
\hline \multirow{4}{*}{0.1} & Biaxial 0/90/0/90 & 1.00 & 1.00 & 1.00 & \\
& Biaxial 0/90/+45/-45 & 0.75 & 0.90 & 0.82 & 0.87 \\
& Biaxial 0/90/90/0 & 0.88 & 0.70 & 0.78 & \\
\hline
\end{tabular}

\section{Conclusions}

The feasibility to differentiate and identify fibre orientation within Biaxial laminate CFRP samples using a combination of commercial-off-the-shelf low temperatures and low cost contact sensors has been demonstrated. The approach centres on the application of and then analysis through Kernel Density Estimates with focus on the low temperature change range within the areas of the sample not directly experiencing heat from a heat source. Such an approach allows for the method to be integrated into existing repair/re-manufacture processes through minor modification to existing setup in the form of temperature sensor placement around a heater mat. The resultant dissipation of transient thermal energy within the structure which can then be analysed to yield accurate indications of fibre orientation. The thermal conductivity of the sample is modulated by the fibre orientation.

Utilising synthetic data representative of samples of double the thickness of physical samples, a non symmetrical but balanced sample of Biaxial 0/90/0/90 continued to produce promising 
indications of high accuracy, recall and precision in true positive identification. This suggests that obtaining experimental data sets from double thickness samples to generate $\overline{K D E} / t$ would improve the identification performance of Biaxial 0/90/90/0 in comparison to the synthetic data. As the approved fibre orientations of aerospace components can be known from the design schematics, and a list of these generated, it is possible to create a $\overline{K D E} / t$ library of fibre orientations that are in use. Should a classification fall outwith the known library, this would provide an indication that the fibre orientation within the structure is not one which is approved and thus should be investigated further.

The method provides a cheap, safe and quick alternative to determine the fibre orientation of composite samples through a routine comparative analysis of KDE plots over a 150 second time period under a $5 \mathrm{~min}$ thermal stimulus at low temperature. Results indicate that the method was also successful in classifying synthetically created noisy data representative of regular and double thickness samples with overall accuracy of 0.93 and 0.87 respectively.

Author Contributions: Conceptualization, D.I.G., R.C.A. and C.T.; methodology, D.I.G., A.W.H. and C.T.; software, D.I.G., A.W.H. and C.T.; validation, D.I.G., A.W.H. and C.T.; formal analysis, D.I.G.; investigation, D.I.G. and A.W.H.; resources, D.I.G., E.J.M. and B.N.; data curation, D.I.G. and E.J.M.; writing-original draft preparation, D.I.G.; writing - review and editing, D.I.G., C.T. and I.A.; visualization, D.I.G. and E.J.M.; supervision, C.T. and I.A.; project administration, D.I.G. and R.C.A.; funding acquisition, D.I.G. and B.N. All authors have read and agreed to the published version of the manuscript.

Funding: This research received no external funding.

Acknowledgments: The Authors would like to thank Collins Aerospace, a Raytheon Technologies company, for the provision of aerospace quality composite samples used in the experimental validation of the method.

Conflicts of Interest: The authors declare no conflict of interest.

\section{Abbreviations}

The following abbreviations are used in this manuscript:

$\begin{array}{ll}\text { CFRP } & \text { Carbon Fibre Reinforced Plastic } \\ \text { FRP } & \text { Fibre Reinforced Plastic } \\ \text { IR } & \text { Infra Red } \\ \text { KDE } & \text { Kernel Density Estimate } \\ \text { MRO } & \text { Maintenance Repair and Overhaul } \\ \text { MSE } & \text { Mean Square Error } \\ \text { OEM } & \text { Original Equipment Manufacturer } \\ \text { PID } & \text { Proportional Integral Derivative } \\ \text { RTD } & \text { Resistence Temperature Detector }\end{array}$

\section{References}

1. Gillespie, D.I.; Hamilton, A.W.; Kruckenberg, T.; Neilson, B; Atkinson, R.C.; Andonovic, I.; Tachtatzis, C. Geometrical Thermal Analysis as a Form of Finite Element Analysis Enhancement. In Advances in Manufacturing Technology XXXIII, Proceedings of the 17th International Conference on Manufacturing Research, Incorporating the 34th National Conference on Manufacturing Research, Queen's University Belfast, Belfast, UK, 10-12 September 2019; Jin, M., Price, Y., Eds.; IOS Press: Amsterdam, The Netherlands, 2019.

2. Uhlmann, E.; Sammler, F.; Richarz, S.; Heitmuller, F.; Bilz, M. Machining of carbon fibre reinforced plastics. In New Production Technologies in Aerospace Industry—5th Machining Innovations Conference (MIC 2014); Elsevier B.V.: Amsterdam, The Netherlands, 2014; Volume 24, pp. 19-24.

3. Ellyin, F.; El-Kadi, H. A fatigue failure criterion for fiber reinforced composite laminae. Compos. Struct. 1990, 15, 61-74. [CrossRef]

4. Pao, Y. Simple Bending Analysis of Laminated Plates by Large-Deflection Theory. J. Compos. Mater. 1970, 4, 380-389. [CrossRef]

5. Ashton, J. Approximate Solutions for Unsymmetrically Laminated Plates. J. Compos. Mater. 1969, 3, $189-191$. [CrossRef] 
6. Hyer, M. Some Observations on the Cured Shape of Thin Unsymmetric Laminates. J. Compos. Mater. 1981, 15, 175-194. [CrossRef]

7. Hou, J.; Jeronimidis, G. Bending stiffness of composite plates with delamination. Compos. Part A Appl. Sci. Manuf. 2000, 31, 121-132. [CrossRef]

8. Rayjade, G.R.; Rao, G.S. Study of Composite Sandwich Structure and Bending Characteristics-A Review. Int. J. Curr. Eng. Technol. 2015, 5, 797-802.

9. Creighton, C.; Sutcliffe, M.; Clyne, T. Multiple field image analysis procedure for characterization of fibre alignment in composites. Compos. Part A Appl. Sci. Manuf. 2001, 32, 221-229. [CrossRef]

10. Altendorf, H.; Jeulin, D. 3D directional mathematical morphology for analysis of fiber orientations. Image Anal. Stereol. 2009, 28, 143-153. [CrossRef]

11. Emerson, M.; Jespersen, K.; Dahl, A.; Conradsen, K.; Mikkelsen, L. Individual fibre segmentation from 3D X-ray computed tomography for characterising the fibre orientation in unidirectional composite materials. Compos. Part A Appl. Sci. Manuf. 2017, 97, 83-92. [CrossRef]

12. Krapez, J.-C.; Gardette, G.; Balageas, D. Thermal Ellipsometry in Steady-State and by Lock-in Thermography. Application to Anisotropic Materials Characterization. Available online: https:/ / www.researchgate.net/publication/234059181_Thermal_ellipsometry_in_steady-state_or_ by_lock-in_thermography_Application_for_anisotropic_materials_characterization (accessed on 9 July 2020).

13. Krapez, J.-C. Thermal Ellipsometry Applied to the Evaluation of Fiber Orientation in Composites; Societe Francaise des Thermiciens, Journee d'Etude sur la Thermographie Quantitative: Paris, France, 7 December 1994.

14. Krapez, J.-C. Thermal Ellipsometry: A Tool Applied for in-Depth Resolved Characterization of Fibre Orientation in Composites. In Review of Progress in Quantitative Nondestructive Evaluation; Springer: New York, NY, USA, 1996; pp. 533-540.

15. Krapez, J.; Gardette, G. Characterisation of anisotropic materials by steady-state and modulated thermal ellipsometry. High Temp. High Press. 1998, 30, 567-574. [CrossRef]

16. Karpen, W.; Wu, D.; Busse, G. A Theoretical Model for the Measurement of Fiber Orientation with Thermal Waves. Res. Nondestruct. Eval. 1999, 11, 179-197. [CrossRef]

17. Fernandes, H.C. Fiber orientation assessment in carbon fiber reinforced composites using infrared thermography. In Proceedings of the 19th International Conference on Composite Materials, Montreal, QC, Canada, 28 July-2 August 2013.

18. Fernandes, H.-C.; Maldague, X. Fiber orientation assessment in complex shaped parts reinforced with carbon fiber using infrared thermography. Quant. Infrared Thermogr. J. 2015, 12, 64-79. [CrossRef]

19. Martens, U.; Schröder, K.-U. Evaluation of infrared thermography methods for analysing the damage behaviour of adhesively bonded repair solutions. Compos. Struct. 2020, 240, 111991. [CrossRef]

20. Florek, M.; Hauser, H. Quantitative data visualization with interactive KDE surfaces. In Proceedings of the SCCG 2010: 26th Spring Conference on Computer Graphics, Budmerice, Slovakia, 13-15 May 2010; ACM Press: New York, NY, USA, 2010; pp. 33-42.

21. Provost, F.; Kohavi, R. Glossary of terms. Mach. Learn. 1998, 30, 271-274. [CrossRef]

Sample Availability: All data underpinning the publication are openly accessible from the University of Strathclyde KnowledgeBase at https:/ / doi.org/10.15129/6240cb56-2742-46b1-a8e9-be8abe342473.

(c) 2020 by the authors. Licensee MDPI, Basel, Switzerland. This article is an open access article distributed under the terms and conditions of the Creative Commons Attribution (CC BY) license (http:// creativecommons.org/licenses/by/4.0/). 